\title{
Orthopedic Principles to Facilitate Enhanced Recovery After Cardiac Surgery
}

Marc W. Gerdisch, MD ${ }^{a, *}$, Keith B. Allen, MD ${ }^{b}$, Yoshifumi Naka, MD, PhD ${ }^{c}$, Mark R. Bonnell, MD ${ }^{d}$, Kevin P. Landolfo, $\mathrm{MD}^{\mathrm{e}}$, John Grehan, MD, PhD ${ }^{f}$, Kendra J. Grubb, MD ${ }^{g}$, David J. Cohen, MD ${ }^{h}$, T. Sloane Guy, MDi', Nirav C. Patel, $M D^{j}$, Vinod H. Thourani, $\mathrm{MD}^{\mathrm{k}}$

\section{KEYWORDS}

- Median sternotomy $\bullet$ Sternal closure $\bullet$ Sternal fixation $\bullet$ Rigid fixation

- Wire cerclage • Sternal wiring • Sternal plating • Sternal bands

\section{KEY POINTS}

- Early patient mobilization is a major driver of recovery after cardiac surgery with open sternotomy.

- Instability of the healing sternal osteotomy hinders postoperative patient movement, causes pain, and puts patients at risk of complications, necessitating restrictive sternal precaution protocols.

- Sternal closure with wire cerclage does not adequately satisfy the three fundamental principles of orthopedic healing: approximation, compression, and rigid fixation.

- Selection of sternal closure methods according to orthopedic principles promotes accelerated recovery and return to normal activities while reducing pain and requirements for opioid analgesics.

\footnotetext{
${ }^{a}$ Department of Cardiothoracic Surgery, Franciscan Health Heart Center, 5255 East Stop 11 Road, Suite 200, Indianapolis, IN 46237, USA; ${ }^{\text {b }}$ Department of Cardiothoracic Surgery, Saint Luke's Mid America Heart Institute, University of Missouri-Kansas City School of Medicine, 4320 Wornall Road, Suite 50-II, Kansas City, MO 64111, USA; ' Division of Cardiothoracic Surgery, Department of Surgery, Columbia University Medical Center, 630 W 168th St, New York, NY 10032, USA; d Department of Cardiothoracic Surgery, University of Toledo, 1735 W Rocket Dr, Toledo, OH 43607, USA; ${ }^{e}$ Department of Cardiothoracic Surgery, Mayo Clinic, 4500 San Pablo Road-Davis 321N, Jacksonville, FL 32224, USA; ${ }^{f}$ Department of Cardiothoracic Surgery, Allina Health, 225 Smith Ave N, Nasseff Specialty Center, Suite 400, St Paul, MN 55102, USA; 9 Department of Cardiothoracic Surgery, Emory University, 550 Peachtree St NE, Davis Fischer 4th Floor, Atlanta, GA 30308, USA; ${ }^{\mathrm{h}}$ Department of Cardiology, Saint Luke's Mid America Heart Institute, University of Missouri-Kansas City School of Medicine, 4320 Wornall Road, Suite 50-II, Kansas City, MO 64111, USA; ${ }^{i}$ Department of Surgery, Division of Cardiac Surgery, Sidney Kimmel Medical College, Thomas Jefferson University, 1025 Walnut St, Suite 607, Philadelphia, PA 19107, USA; ${ }^{j}$ Department of Cardiothoracic Surgery, Lenox Hill Hospital, 130 East 77th Street, 4th Floor, New York, NY 10075, USA; ${ }^{k}$ Department of Cardiovascular Surgery, Marcus Heart and Vascular Center, Piedmont Heart Institute, 95 Collier Road, Suite 5015, Atlanta, GA 30309, USA

* Corresponding author.

E-mail address: mgerdisch@openheart.net
} 


\section{THE EVOLUTION FROM “FAST-TRACK" TO “ENHANCED RECOVERY" AFTER CARDIAC SURGERY}

Around the world, clinical and economic analyses increasingly show that evidencebased initiatives to improve and shorten the course of in-hospital recovery positively affect quality of care, patient outcomes, and cost-effectiveness of care. ${ }^{1-4}$ In Europe, the Enhanced Recovery After Surgery (ERAS) Society has formalized the movement to promote improvements in postsurgical recovery of patients by releasing a series of guidelines for multimodal, transdisciplinary care improvement. ${ }^{1}$ Early successes improving recovery times and clinical outcomes after colorectal surgery have served as a model for the development of similar initiatives in gynecologic, bariatric, gastrointestinal, liver, and head and neck surgery. ${ }^{1}$

The general principles that have been formalized in current ERAS guidelines have been informally used in cardiac surgery for at least 3 decades. As early as 1990, Krohn and colleagues ${ }^{5}$ verified that proactive prevention and prompt correction of noncardiac complications after surgery shortened hospital length of stay (LOS) and resulted in fewer readmissions. In 1994, Engelman and colleagues ${ }^{6}$ found that a fast-track recovery protocol aimed at reducing complications and accelerating discharge was associated with statistically significant and clinically meaningful decreases in time to extubation, peak weight gain, intensive care unit (ICU) LOS, and overall hospital LOS without increases in morbidity or mortality.

Subsequent studies in both Europe and the United States built on these successes by gradually adding more ERAS principles to the perioperative process. ${ }^{7}$ Zaouter and colleagues $^{8}$ (France) examined extubation outcomes and postoperative complications in 38 patients undergoing robotic, totally endoscopic coronary artery bypass grafting (CABG), managed according to an ERAS-guided protocol, compared with a matched cohort undergoing conventional CABG with traditional perioperative management. They found that patients in the robotic surgery/ERAS group could be safely extubated in the operating room, experienced lower transfusion rates, experienced shorter ICU stays (mean reduction of 24 hours), and experienced overall hospital stays (mean reduction of 4 days) compared with patients managed with standard care $(P<.05)$. A notable variable in these procedures was the use of minimally invasive, sternum-sparing approaches in the robotic surgery group; the critical role of a stable sternum in the healing process is central to the present discussion.

In 2016, Fleming and colleagues ${ }^{9}$ from the United Kingdom published the results of a prospective, observational study comparing outcomes in 105 consecutive adult patients undergoing any scheduled, elective cardiac surgery, before and after implementation of a perioperative care bundle based on ERAS principles. The aim was to evaluate whether small practice improvements distributed throughout the course of perioperative management could accumulate to result in meaningful gains in clinical outcomes. Even in this small study, the group managed according to ERAS principles $(n=52)$ experienced less postoperative morbidity and mortality and reported improved postoperative pain scores compared with patients who underwent surgery before the care bundle was implemented.

A clinical trial carried out in China and published in 2018 by Li and colleagues ${ }^{10}$ evaluated outcomes after randomization to either an ERAS protocol or standard of care in 226 patients undergoing elective cardiac value procedures, with or without cardiac ablation and/or CABG. The study found that patients in the ERAS group spent significantly less time on mechanical ventilation, had a shorter stay in the ICU, and were ready for discharge a day earlier. Overall cost of care was also significantly reduced in the ERAS patients. 
In 2019, Grant and colleagues ${ }^{11}$ published a retrospective analysis of prospectively collected data from 451 consecutive patients undergoing CABG, valvular, or combination procedures, as part of a stepwise implementation of an institutional early-recovery program for cardiac surgery at their hospital. After stratifying patients into low- and high-compliance groups (based on how many protocol measures were followed) and then propensity-matching patients between groups, they found that implementation of their program significantly improved early extubation rates and hospital LOS.

Most recently, Williams and colleagues ${ }^{12}$ published the results of a large feasibility study of prospectively collected, retrospectively reviewed data comparing outcomes in 443 US patients managed according to an ERAS-based protocol, versus 489 managed according to traditional standard of care. Standardized processes included preoperative patient education, carbohydrate loading 2 hours before general anesthesia, multimodal opioid-sparing analgesia, goal-directed perioperative insulin infusion, a rigorous bowel regimen, and early mobilization. ${ }^{13}$ One-year clinical data and survey results showed ERAS-based patient management was associated with reduced median overall LOS ( 6 vs 7 days; $P<.01$ ), reduced ICU time (28 vs 43 hours; $P<.01)$, lower rate of gastrointestinal complications $(3.6 \%$ vs $6.8 \% ; P<.05)$, and an $8 \mathrm{mg}$ morphine equivalent reduction in opioid use in the first 24 hours postoperatively $(P<.01)$. Patient satisfaction was also higher in the ERAS-managed group.

Based on the growing body of evidence regarding the individual practices that benefit recovery and outcomes after cardiac surgery, the ERAS Cardiac Society was formed in 2017 to develop a set of recommendations for the field. The founding group of cardiac surgeons, anesthesiologists, and intensivists published its first, expert-consensus review of evidence-based practices for Enhanced Recovery After Cardiac Surgery in 2019, in partnership with the ERAS Society. ${ }^{14}$

\section{MEDIAN STERNOTOMY AS AN OBSTACLE AND OPPORTUNITY FOR ERAS AFTER CARDIAC SURGERY}

ERAS guidelines across all disciplines are typically organized into preoperative, intraoperative, and postoperative strategies, each ranked by strength of recommendation (based on expected benefits vs potential harms) and by the quality of evidence underlying each recommendation. The Enhanced Recovery After Cardiac Surgery Society (ERAS Cardiac) guidelines are evidence based and are similar to those of other disciplines. ${ }^{14}$ Recommended preoperative measures include optimization of glycemic control, kidney function, and nutrition; patient education; and cessation of smoking and hazardous alcohol consumption. Intraoperatively, the guidelines recommend use of protocols to reduce surgical site infections, avoidance of hyperthermia during patient rewarming on cardiopulmonary bypass, use of tranexamic acid or epsilon aminocaproic acid for bleeding management during on-pump cardiac surgical procedures, and use of rigid-plate fixation (RPF) to provide maximal stability to the healing osteotomy and potentially reduce the incidence of major sternal complications. Postoperative recommendations include continued perioperative management of glycemic control and kidney function; maintenance of normothermia, chest tube patency, and thromboprophylaxis; multimodal, opioid-sparing pain management; goal-directed fluid therapy; systematic screening for delirium; and extubation within 6 hours of surgery.

The ERAS Cardiac guidelines differ from those for other disciplines. First, the ERAS Cardiac recommendations do not emphasize use of minimally invasive surgical (MIS) approaches. Although MIS parasternal and minithoracotomy approaches have been developed for single-valve operations and select, combined cardiac surgical procedures, median sternotomy remains the most common incision for open heart surgery. ${ }^{15}$ 
Although it is an invasive procedure, median sternotomy provides access to every part of the heart and the large blood vessels within the chest. It is, therefore, likely to remain a common surgical approach for the foreseeable future. ${ }^{16}$

Second, the ERAS Cardiac guidelines include no specific recommendations for early postoperative enteral feeding and mobilization, even though these measures are known to work together to stimulate resumption of gut function, preserve musculoskeletal function, and promote a faster and safer return to normal activities after surgery. ${ }^{17-22}$ A sternotomy incision limits early mobilization and rehabilitation activities. Although the ERAS Cardiac guidelines do recommend that cardiac surgery programs develop multidisciplinary institutional guidelines to address early feeding and mobilization, ${ }^{14}$ traditional sternal precautions prioritize the stability of the bony union over patient mobility, greatly limiting opportunities for return to normal activity and physical rehabilitation in the postoperative recovery period. ${ }^{17,18,23}$ The authors propose that the limitations that sternal healing imposes on early mobilization and physical activity during recovery represent a potential target for further gains in the pace of recovery and improved outcomes after cardiac surgery.

\section{ORTHOPEDIC PRINCIPLES: THE RECIPE FOR SUCCESSFUL HEALING AND EARLY MOBILIZATION AFTER STERNOTOMY}

Healing of osseous fractures, whether traumatic or as a result of surgical osteotomy or osteochondrotomy, depends on a complex interaction between biological processes and biomechanical forces. ${ }^{24-27}$ The biomechanical principles that promote successful healing include accurate reapproximation, alignment, and reduction of the osteotomy gap to foster restoration of blood flow and cellular communication; compression that is adequate to encourage osteosynthesis but not so excessive as to impede blood flow; and rigid fixation of the union to stabilize it against movement and prevent recurring microfractures. ${ }^{24-27}$

Stability of the sternum promotes revascularization and bone formation. In contrast, instability in the presence of repetitive distractive tensile loads and shear forces allows bone-on-bone movement within the osteotomy. Such movement promotes the formation of a fibrocartilaginous callous that must later remodel into ossified bone, or it may inhibit the healing process altogether. ${ }^{25,28}$

Like all other bone fractures, the sternotomy is governed by these same orthopedic principles and biological processes. It is also subject to continuous forces from breathing, coughing, movements of the head, spine, and upper extremities, and ambulation. A critical role of the healthy sternum is to provide flexible support and central communication and distribution of the forces from these activities. ${ }^{28}$ Sternal instability after closure is closely associated with poor sternal healing, deep sternal wound infection (DSWI), and sternal dehiscence or nonunion, as well as postoperative pain and increased requirement for analgesia. ${ }^{29,30}$ These sternal complications represent major drivers of postsurgical morbidity and mortality after cardiac surgery, especially in high-risk patients. The incidence of sternal wound infection is as high as $5 \%$, and mediastinitis has been reported to occur in $0.8 \%$ to $2.3 \%$ of patients. ${ }^{31} \mathrm{DSWI}$ in particular is associated with as much as a 4-fold increase in 1-year postoperative mortality, 3-fold increase in length of hospital stay, and 2.5-fold higher cost of care compared with patients without infection. ${ }^{32}$

Even though early postoperative mobilization and resumption of physical activities are known to be advantageous to healing of bone fractures and to functional recovery in general, ${ }^{18,23,26}$ cardiothoracic surgeons face a dilemma in choosing methods for sternal closure, governed by the effort to balance 2 potential risks: one, that patient 
movements and consequent forces on an unstable sternal union will disrupt healing and invite infection; and two, that devices providing rigid fixation represent an obstacle to emergent sternal reentry. ${ }^{33}$

Newer techniques and devices for closure, coupled with higher-quality evidence regarding their relative ability to stabilize the sternum for optimal healing, suggest a path toward reducing the influence of sternal healing as a limiting factor on the ERAS principle of early mobilization and return to normal activity.

\section{STERNAL-CLOSURE TECHNIQUES IN THE CONTEXT OF ORTHOPEDIC PRINCIPLES Wire Cerclage}

Traditional wire cerclage remains by far the most common technique for sternal closure after median sternotomy, ${ }^{16}$ leaving cardiac surgeons as the only specialty that still relies on wire cerclage for postosteotomy fixation. In this technique, the cut edges of the sternal osteotomy are brought together at 5 to 8 levels with lengths of stainless-steel surgical wire, wrapped around or through the bone and tightened (Fig. 1). This technique is simple and inexpensive to implement, and in case of emergent need for sternal reentry, the wires can readily be cut for removal.

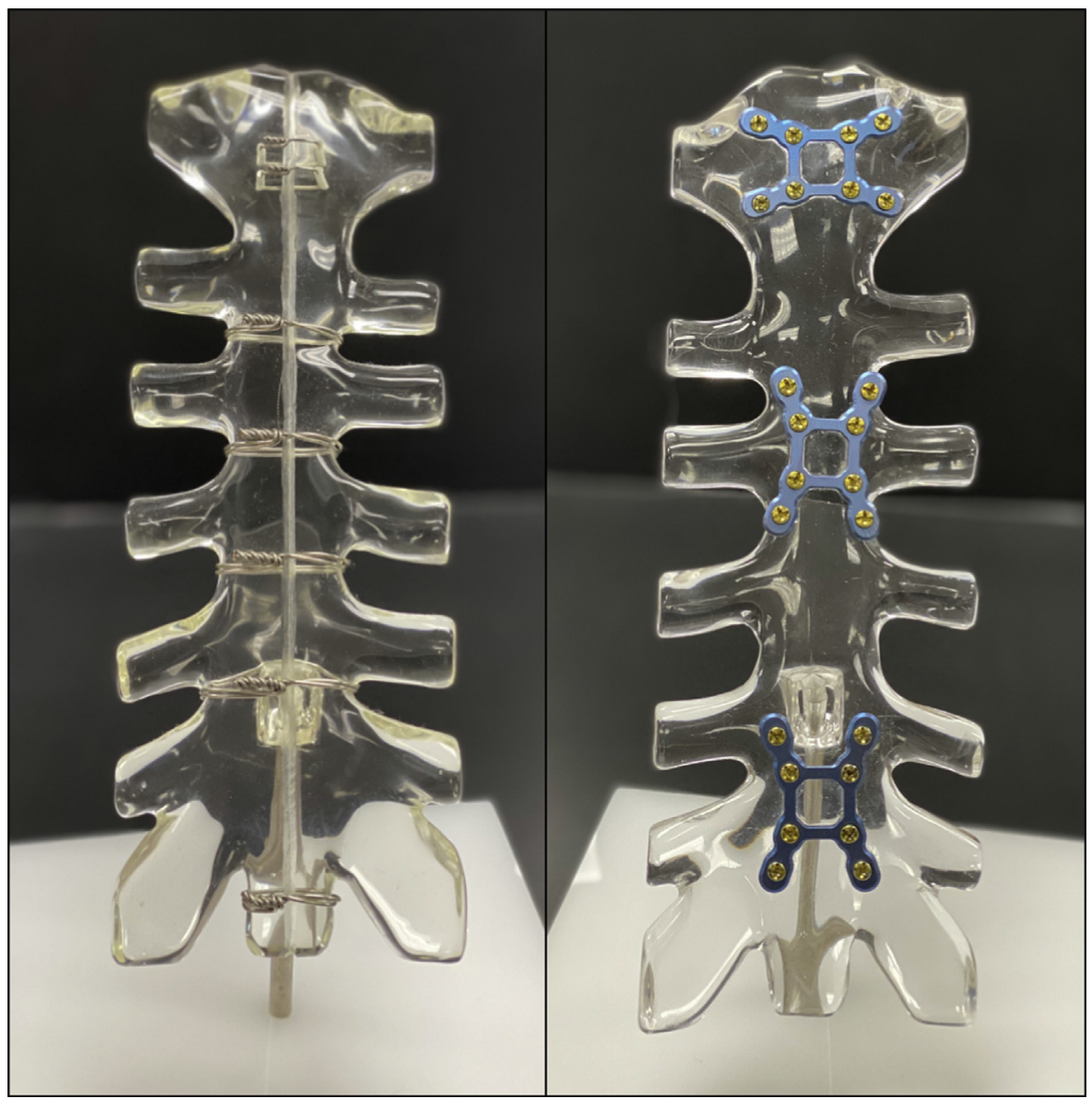

Fig. 1. Wire cerclage (left) and RPF (right). Photographs provided by Zimmer Biomet. 
Wire cerclage provides for 2 of the 3 essential requirements for sternal union, namely, approximation and compression. ${ }^{34}$ However, it has been shown that wire cerclage provides, at best, suboptimal fixation and stabilization of the healing bone. ${ }^{28}$ It is also notable that the low ratio of surface-area contact between wire and bone concentrates the compressive forces along the wires, with the potential for notching or cutting of the bone and even complete transverse sternal fracture. ${ }^{28}$ Although alternative techniques for single-wire cerclage, such as double-wire, figure-of-8, and Robicsek's weave, have been developed to improve the relative distribution of compressive forces, ${ }^{16,35}$ they have not been shown to reduce rates of sternal dehiscence or DSWI compared with single wires. ${ }^{36,37}$ Furthermore, despite providing a range of compressive forces, none of these approaches address the biomechanical problem of inadequate fixation. ${ }^{35}$ Rigid Fixation is especially critical in patients who are considered to be high risk because of morbid obesity, chronic obstructive pulmonary disease, diabetes mellitus, or other comorbidities, because sternal dehiscence is associated with a markedly higher mortality in these patients. ${ }^{30}$

\section{Steel Bands}

Stainless-steel bands have been explored as a potential adjunct to wires, chiefly because their wider profile provides for improved contact surface area between the band and bone, thus potentially reducing wire pull-through and preventing transverse fractures as the mechanism for failure. Three studies have found that the use of steel bands in combination with standard wiring reduces the risk of sternal dehiscence compared with single steel wires used alone. ${ }^{38-40}$ However, none of the studies found that steel bands reliably reduced the incidence of mediastinitis.

Criticisms of steel bands include that their structural rigidity prevents them from conforming to the patient-specific shape of the sternum. The bands also do not have a mechanism to lock them in place after tightening, and they may be difficult to remove in case of the need for sternal reentry. ${ }^{16}$ Furthermore, although steel bands adequately satisfy the requirements for approximation and compression, the directional forces are similar to wires and do not provide rigid fixation.

\section{Polymer Cable Ties}

A newer alternative to steel wires or bands is cable ties made of polyether ether ketone (PEEK), a high-performance, biocompatible, nickel-free polymer that has been used since the 1990s to replace metal in a growing number of implanted medical devices. ${ }^{41}$ The polymer cable ties work according to a principle similar to steel bands, but are more flexible and malleable. ${ }^{41}$ The recommended use is to place 5 ties along the sternum (the first transsternally through the manubrial bone, 3 peristernal bands through the intercostal spaces, and the fifth transsternally through the xiphoid region). The free end of each tie is passed through the locking head at the other end, tightened by hand to ensure accurate sternal approximation, and then tensioned to a force of $200 \mathrm{~N}$ using a system-specific application device, which also cuts away excess tie material. As with steel bands, the cable ties are wider than surgical wire $(4.2 \mathrm{~mm}$ vs $0.7 \mathrm{~mm}$ for US Pharmacopeia 5 steel wire) and thus provide increased area of contact between implant and bone, potentially reducing the risk of sternal damage. In support of this idea, PEEK-based cable ties have been shown, in engineering studies, to have equivalent or better static-loading strength, fatigue strength, and resistance to bone cutthrough compared with stainless-steel surgical wire. ${ }^{42,43}$

A commercially available polymer sternal cable-tie system has been evaluated in several studies, with mixed results. ${ }^{41,44-47}$ A retrospective comparison of 95 sternotomies closed with polymer cable ties versus 498 closed with transverse, interrupted 
figure-of-8 stainless-steel wires found no difference in superficial or DSWI between groups. ${ }^{47}$ Another retrospective, nonrandomized comparison found that DSWI occurred in $2.6 \%$ (8/309) of patients who underwent sternal closure with surgical wires alone versus no occurrences $(0 / 300)$ in patients closed with a combination of surgical wires and polymer cable ties. ${ }^{46}$

A 118-patient, prospective, randomized study found no difference in pain-related outcomes but more sternal and manubrial movement, by ultrasound, when polymer cable ties were used instead of steel wires. In contrast, a larger randomized trial found significantly lower pain scores and rates of sternal dehiscence with the use of cable ties, with similar rates of infection (2.76\%). ${ }^{48}$ Independent criticism of these conflicting studies has noted that both are limited by lack of definition/standardization of wiring technique, different choice of outcomes, and biases in study design and reporting. ${ }^{49,50}$ Given further conflicting results in biomechanical studies comparing conventional versus figure-of- 8 wire cerclage, polymer cable ties, and steel bands, ${ }^{42,43}$ higher-quality data are required before definitive conclusions can be reached about the appropriate role of polymer cable ties in the sternal closure toolkit. Of key importance, despite their proposed advantages over wires and bands, polymer cable ties also do not provide orthopedic stabilization of the osteotomy and are not considered rigid fixation.

\section{Rigid-Plate Fixation}

Sternal fixation with rigid plates, mounted to the sternum with screws (see Fig. 1), is specifically noted in the ERAS Cardiac guidelines for its potential to "be useful to improve or accelerate sternal healing and reduce mediastinal wound complications." 14 This guideline is presented as a recommendation with moderate benefit (class Ila), based on evidence from one or more randomized clinical trial (level B-R), as well as from biomechanical studies.

Experimentally, rigid fixation with sternal plates has been shown to reduce the extent of sternal gap development under static load compared with wire cerclage. ${ }^{28,51}$ A technique for rigid-plate sternal fixation in humans was first published by Gottlieb and colleagues ${ }^{52}$ in 1994 using plates intended for mandibular fixation. Later, Song and colleagues ${ }^{33}$ published a report evaluating a commercially available plate system configured specifically for the sternum in 45 patients, each of whom had a minimum of 3 established risk factors for dehiscence and mediastinitis. This system consisted of multiple configurations of titanium plates secured with bicortical screws of various sizes depending on the patient's specific anatomy. Compared with matched controls closed with wire cerclage, these patients experienced significantly lower rates of mediastinitis $(14.8 \%$ vs $0 \%, P=.006)$. Use of this system also eliminated emergent sternal reentry as a major obstacle to surgeon comfort with use of rigid plates, because it was designed to be cut easily with wire cutters standard to the operating room. ${ }^{33}$

Since those early reports, the results of several studies of both observational and randomized, controlled designs have been published showing that RPF is associated with earlier extubation, ${ }^{53}$ shorter postoperative LOS, ${ }^{53-55}$ improved sternal healing, ${ }^{34,56,57}$ reduced incidence of early sternal wound complications, mediastinitis and DSWI, ${ }^{34,54,57-60}$ less pain and lower utilization of analgesic and narcotic medications, ${ }^{55,56,58,60,61}$ less radiographic evidence of sternal displacement during recovery, ${ }^{55}$ and similar or lower overall cost of care. ${ }^{34,59,61}$ The benefits may especially be significant in patients at high risk of sternal dehiscence. ${ }^{62}$

A recent, prospective, single-blinded, multicenter, randomized trial compared outcomes in patients undergoing median sternotomy for cardiac surgery at 12 US sites, 
and closed either with rigid plates $(n=116)$ or wire cerclage $(n=120) .{ }^{34}$ Importantly, the study excluded high-risk patients in order to understand the potential benefits of RPF for the general cardiac surgery population. For the primary endpoint of the study, radiographic and clinical evidence of sternal healing, RPF resulted in better sternal healing scores at 3 and 6 months compared with wire cerclage $(P<.0001$ and $P=.0007$, respectively). Sternal union rates were also greater at these time points $(P<.0001$ and $P=.03)$, with no sternal complications at 6 months in the RPF group versus $5 \%$ in the wire cerclage group $(P=.03)$. Overall cost of care was neutral between groups over the total study period.

Furthermore, Allen and colleagues ${ }^{61}$ noted a greater proportion of patients treated with RPF reported absence of pain after coughing at 3 and 6 weeks of follow-up $(P=.001$ and $P=.005)$, and at rest at 6 weeks and 3 months $(P=.02$ and $P=.03)$ compared with wire cerclage. These scores correlated with better sternal healing. Patients in the RPF group also reported significantly better quality of life at 3 weeks, 6 weeks, and 6 months. Additional cost analysis using a 90-day global payment model confirmed RPF improved patient outcomes at both 90 and 180 days without increasing cost to the health care system. These results were in patients with standard risk factors for cardiac surgery and illustrate that RPF is likely to benefit all sternotomy patients regardless of their risk profile.

A potential concern for surgeons new to the use of RPF is whether the additional foreign material from the plates could result in residual dead space above the sternum after closure, and therefore, more superficial wound issues, compared with wiring. Clinical experience has shown that this potential problem can be readily addressed by closure technique. When closing fascia after RFP, bites should be taken so that the suture goes under the plates, thus pulling the fascia down to the plates and obliterating any dead space.

\section{Other Devices and Methods for Sternal Fixation}

An assortment of other devices, such as a cabling and plating system, transverse bridging plates, locking plates and screws, j-hook plates, quick-release clamps, and special clasps, have been proposed or developed for sternal closure. Many of these products do not incorporate the orthopedic principle of rigid fixation and lack sufficient randomized evidence to be relevant to this review. In addition, chemical and biologic enhancement of healing using bone cement and other polymerizing agents, resorbable materials, platelet-rich plasma, and hydrogels have been proposed to encourage osteosynthesis; however, these products do not provide rigid fixation, and their use as an adjunct to rigid fixation has not been studied. The authors refer the reader to several excellent reviews that go into greater depth regarding the full spectrum of products and techniques under investigation. ${ }^{28}$

Based on the available evidence, the ERAS Cardiac working group concluded that RPF should be the recommended method of closure after median sternotomy, with benefits that would especially accrue to patients whose comorbidities put them at high risk of sternal dehiscence. ${ }^{14}$

\section{RIGID-PLATE FIXATION AND RAPID MOBILIZATION}

The paradigm for managing postsurgical recovery is changing across surgical disciplines, informed by emphasis on evidence over tradition. 1,7,63-65 Evidence shows that deliberate, strategic interventions aimed at safely getting the patient extubated, awake, eating, moving, and home as soon as possible after surgery have positive 
benefits for the patient's physical, mental, and psychosocial recovery as well as for cost-effective health care delivery. ${ }^{1-4,7,63-65}$

In cardiac surgery, the ERAS Cardiac recommendation for use of RPF to establish sternal stability and promote healing after median sternotomy has enabled a shift in attitudes toward sternal precautions. Historically, physical rehabilitation programs have called for drastic restrictions on activity and upper-body mobility, dictated by the limitations imposed by patient pain and the slow pace of healing associated with the unstable, wired sternum. ${ }^{17,18,20,22,23}$ As real-world experience with RPF proves consistent with controlled trials, surgeons are piloting new paths to patient recovery. For example, following 2 years of study participation and data review, in March 2017, Franciscan Health Heart Center (Indianapolis, Indiana, USA) made RPF the standard closure method for all sternotomies. The departments of Cardiac Surgery and Physical Therapy developed a rapid assessment and mobilization protocol (Table 1). This aggressive protocol was made possible by stable sternotomies allowing for near-normal upper body mobility. Sternal stability has dramatically diminished pain, and after discharge from ICU, $70 \%$ of patients do not receive opioids, including after discharge. Patients are permitted to use their arms immediately after surgery with early freedom to get out of bed, up from a chair, or off the commode unassisted. Patients are now much more likely to be discharged home instead of to a skilled nursing facility, and to return to normal activities and back to work earlier (see Table 1).

\begin{tabular}{|ll}
\hline $\begin{array}{l}\text { Table } 1 \\
\text { Example of accelerated mobilization protocol for patients with rigid plate fixation }\end{array}$ \\
\hline Timing & Allowed Activities \\
\hline At discharge & - Ride in a car \\
& - Walk inside house or in yard \\
& - Climb 1 flight of stairs (no carrying) \\
\hline First week & - Lift 10 pounds each hand \\
& - Light household activities: limited cooking, \\
& dishwashing, dusting, small repairs \\
\hline $1-2 \mathrm{wk}$ & - Attend religious services \\
& - Go out to eat or going to movies \\
& - Visit friends \\
\hline $2-3 \mathrm{wk}$ & - Take out trash \\
& - Grocery shopping \\
& - Mow lawn with a riding mower \\
& - Putt golf balls in yard \\
& - Evaluation for driving a car at 2-wk office visit \\
\hline $2-4 \mathrm{wk}$ & - Sexual relations \\
\hline $4-8 \mathrm{wk}$ & - Lift 15 pounds each hand (most restrictions \\
& lifted by 5 wk) \\
& - Heavy household activities: vacuuming, sweeping, \\
& mopping, changing bed linens \\
& - Gardening or pulling weeds \\
& - Mow lawn with a push mower \\
\hline $8-10 \mathrm{wk}$ & Bowling \\
& - Folfing \\
\hline
\end{tabular}

Table created following guidelines of Franciscan Health Heart Center, Departments of Cardiothoracic Surgery and Physical Therapy. 
In addition to reducing all forms of sternal wound complications, RPF removes most limitations following sternotomy. Mobilization following RPF is conceptually similar to that for patients treated by minimally invasive approaches, without full sternotomy incisions. Indeed, evidence from studies of recovery after robotic, transcatheter, and other minimally invasive cardiac-surgical procedures supports the assertion that eliminating concern for sternal instability in the postoperative phase of care improves recovery, patient experience, and cost-effectiveness. ${ }^{8,66-70}$

\section{SUMMARY}

Evidence supporting best practices throughout the perioperative process has fostered significant changes in attitudes toward postoperative recovery, across a wide range of surgical disciplines. Programs aimed at promoting structured, rapid physical recovery, with the earliest-possible safe return to normal activities have yielded improvements in clinical, economic, and patient-reported outcomes, representing a win-win-win scenario for all stakeholders. Median sternotomy presents specific challenges for recovery after cardiac surgery. However, adoption of evidence-based techniques, including sternal RPF, maximizes adherence to orthopedic principles, minimizes patient pain, reduces opioid use, promotes healing, and can enable downstream implementation of aggressive, rapid mobilization protocols that are consistent with ERAS principles.

\section{ACKNOWLEDGMENTS}

Jeanne McAdara, PhD (Biolexica Health Science Communications, Longmont, Colorado) provided professional medical writing assistance. The medical writer worked at the direction of the authors.

\section{DISCLOSURE}

MWG discloses a consulting relationship with Zimmer Biomet; KBA discloses a research grant and consulting relationship with Zimmer Biomet; David Cohen, MD, discloses research funding for health economics core laboratory with Zimmer Biomet. All other authors have nothing to disclose with regard to commercial support. The authors maintained full control over this manuscript.

\section{REFERENCES}

1. Ljungqvist $O$, Scott $M$, Fearon KC. Enhanced recovery after surgery: a review. JAMA Surg 2017;152(3):292-8.

2. Spanjersberg WR, Reurings J, Keus F, et al. Fast track surgery versus conventional recovery strategies for colorectal surgery. Cochrane Database Syst Rev 2011;(2):CD007635.

3. Stone AB, Grant MC, Pio Roda C, et al. Implementation costs of an enhanced recovery after surgery program in the United States: a financial model and sensitivity analysis based on experiences at a quaternary academic medical center. J Am Coll Surg 2016;222(3):219-25.

4. Thiele RH, Rea KM, Turrentine FE, et al. Standardization of care: impact of an enhanced recovery protocol on length of stay, complications, and direct costs after colorectal surgery. J Am Coll Surg 2015;220(4):430-43.

5. Krohn BG, Kay JH, Mendez MA, et al. Rapid sustained recovery after cardiac operations. J Thorac Cardiovasc Surg 1990;100(2):194-7.

6. Engelman RM, Rousou JA, Flack JE, et al. Fast-track recovery of the coronary bypass patient. Ann Thorac Surg 1994;58(6):1742-6. 
7. Noss C, Prusinkiewicz C, Nelson G, et al. Enhanced recovery for cardiac surgery. J Cardiothorac Vasc Anesth 2018;32(6):2760-70.

8. Zaouter C, Imbault J, Labrousse L, et al. Association of robotic totally endoscopic coronary artery bypass graft surgery associated with a preliminary cardiac enhanced recovery after surgery program: a retrospective analysis. J Cardiothorac Vasc Anesth 2015;29(6):1489-97.

9. Fleming 1O, Garratt C, Guha R, et al. Aggregation of marginal gains in cardiac surgery: feasibility of a perioperative care bundle for enhanced recovery in cardiac surgical patients. J Cardiothorac Vasc Anesth 2016;30(3):665-70.

10. Li M, Zhang J, Gan TJ, et al. Enhanced recovery after surgery pathway for patients undergoing cardiac surgery: a randomized clinical trial. Eur J Cardiothorac Surg 2018;54(3):491-7.

11. Grant MC, Isada T, Ruzankin P, et al. Results from an enhanced recovery program for cardiac surgery. J Thorac Cardiovasc Surg 2020;159(4):1393-402.e7.

12. Williams JB, McConnell G, Allender JE, et al. One-year results from the first USbased enhanced recovery after cardiac surgery (ERAS Cardiac) program. J Thorac Cardiovasc Surg 2019;157(5):1881-8.

13. McConnell G, Woltz P, Bradford WT, et al. Enhanced recovery after cardiac surgery program to improve patient outcomes. Nursing 2018;48(11):24-31.

14. Engelman DT, Ben Ali W, Williams JB, et al. Guidelines for perioperative care in cardiac surgery: enhanced recovery after surgery society recommendations. JAMA Surg 2019;154(8):755-66.

15. Dalton ML, Connally SR, Sealy WC. Julian's reintroduction of Milton's operation. Ann Thorac Surg 1992;53(3):532-3.

16. Nenna A, Nappi F, Dougal J, et al. Sternal wound closure in the current era: the need of a tailored approach. Gen Thorac Cardiovasc Surg 2019;67(11):907-16.

17. Adams J, Lotshaw A, Exum E, et al. An alternative approach to prescribing sternal precautions after median sternotomy, "Keep Your Move in the Tube". Proc (Bayl Univ Med Cent) 2016;29(1):97-100.

18. El-Ansary D, LaPier TK, Adams J, et al. An evidence-based perspective on movement and activity following median sternotomy. Phys Ther 2019;99(12):1587-601.

19. Katijjahbe MA, Granger CL, Denehy L, et al. Standard restrictive sternal precautions and modified sternal precautions had similar effects in people after cardiac surgery via median sternotomy ('SMART' Trial): a randomised trial. J Physiother 2018;64(2):97-106.

20. Katijjahbe MA, Denehy L, Granger CL, et al. The Sternal Management Accelerated Recovery Trial (S.M.A.R.T) - standard restrictive versus an intervention of modified sternal precautions following cardiac surgery via median sternotomy: study protocol for a randomised controlled trial. Trials 2017;18(1):290.

21. Balachandran S, Lee A, Royse A, et al. Upper limb exercise prescription following cardiac surgery via median sternotomy: a web survey. J Cardiopulm Rehabil Prev 2014;34(6):390-5.

22. Cahalin LP, Lapier TK, Shaw DK. Sternal precautions: is it time for change? Precautions versus restrictions - a review of literature and recommendations for revision. Cardiopulm Phys Ther J 2011;22(1):5-15.

23. Pengelly J, Pengelly M, Lin KY, et al. Resistance training following median sternotomy: a systematic review and meta-analysis. Heart Lung Circ 2019;28(10): 1549-59.

24. Marsell R, Einhorn TA. The biology of fracture healing. Injury 2011;42(6):551-5.

25. Sathyendra V, Darowish M. Basic science of bone healing. Hand Clin 2013;29(4): 473-81. 
26. Dabis J, Templeton-Ward O, Lacey AE, et al. The history, evolution and basic science of osteotomy techniques. Strateg Trauma Limb Reconstr 2017;12(3): 169-80.

27. Ilizarov GA. Transosseous osteosynthesis: theoretical and clinical aspects of the regeneration and growth of tissue. Berlin (Germany): Springer Science \& Business Media; 2012.

28. Gandhi HS. Rationale and options for choosing an optimal closure technique for primary midsagittal osteochondrotomy of the sternum, part 2: a theoretical and critical review of techniques and fixation devices. Crit Rev Biomed Eng 2019; 47(1):27-57.

29. Raman J, Straus D, Song DH. Rigid plate fixation of the sternum. Ann Thorac Surg 2007;84(3):1056-8.

30. Balachandran S, Lee A, Denehy L, et al. Risk factors for sternal complications after cardiac operations: a systematic review. Ann Thorac Surg 2016;102(6): 2109-17.

31. Imren $Y$, Selek $H$, Zor $H$, et al. The management of complicated sternal dehiscence following open heart surgery. Heart Surg Forum 2006;9(6):E871-5.

32. Yusuf E, Chan M, Renz N, et al. Current perspectives on diagnosis and management of sternal wound infections. Infect Drug Resist 2018;11:961-8.

33. Song DH, Lohman RF, Renucci JD, et al. Primary sternal plating in high-risk patients prevents mediastinitis. Eur J Cardiothorac Surg 2004;26(2):367-72.

34. Allen KB, Thourani VH, Naka Y, et al. Randomized, multicenter trial comparing sternotomy closure with rigid plate fixation to wire cerclage. J Thorac Cardiovasc Surg 2017;153(4):888-96.e1.

35. Losanoff JE, Collier AD, Wagner-Mann CC, et al. Biomechanical comparison of median sternotomy closures. Ann Thorac Surg 2004;77(1):203-9.

36. Vos RJ, Van Putte BP, Kloppenburg GTL. Prevention of deep sternal wound infection in cardiac surgery: a literature review. J Hosp Infect 2018;100(4):411-20.

37. Pinotti KF, Cataneo DC, Rodrigues OR, et al. Closure of the sternum with anchoring of the steel wires: systematic review and meta-analysis. J Thorac Cardiovasc Surg 2018;156(1):178-86.

38. Franco S, Herrera AM, Atehortúa M, et al. Use of steel bands in sternotomy closure: implications in high-risk cardiac surgical population. Interact Cardiovasc Thorac Surg 2009;8(2):200-5.

39. Bhattacharya S, Sau I, Mohan M, et al. Sternal bands for closure of midline sternotomy leads to better wound healing. Asian Cardiovasc Thorac Ann 2007;15(1): 59-63.

40. Riess FC, Awwad N, Hoffmann B, et al. A steel band in addition to 8 wire cerclages reduces the risk of sternal dehiscence after median sternotomy. Heart Surg Forum 2004;7(6):387-92.

41. Grapow MT, Melly LF, Eckstein FS, et al. A new cable-tie based sternal closure system: description of the device, technique of implantation and first clinical evaluation. J Cardiothorac Surg 2012;7:59.

42. Orhan SN, Ozyazicioglu MH. Evaluation of sternum closure methods by means of a nonlinear finite element analysis. Proc Inst Mech Eng H 2019;233(12):1282-91.

43. Orhan SN, Ozyazicioglu MH, Colak A. A biomechanical study of 4 different sternum closure techniques under different deformation modes. Interact Cardiovasc Thorac Surg 2017;25(5):750-6.

44. Marasco SF, Fuller L, Zimmet A, et al. Prospective, randomized, controlled trial of polymer cable ties versus standard wire closure of midline sternotomy. J Thorac Cardiovasc Surg 2018;156(4):1589-95.e1. 
45. Samuels L. Sternal closure with tie bands: a word of caution. Ann Thorac Surg 2016;102(2):e121-2.

46. Stelly MM, Rodning CB, Stelly TC. Reduction in deep sternal wound infection with use of a peristernal cable-tie closure system: a retrospective case series. J Cardiothorac Surg 2015;10:166.

47. Melly L, Gahl B, Meinke R, et al. A new cable-tie-based sternal closure device: infectious considerations. Interact Cardiovasc Thorac Surg 2013;17(2):219-23 [discussion: 223].

48. Nezafati P, Shomali A, Kahrom M, et al. ZipFix versus conventional sternal closure: one-year follow-up. Heart Lung Circ 2019;28(3):443-9.

49. Omer S. Polymer cable tie closure of the sternum: is it an acceptable fix. J Thorac Cardiovasc Surg 2018;156(4):1611-2.

50. Tam DY, Fremes SE. Cable ties for chest closure: ZipFix or ZipFail. J Thorac Cardiovasc Surg 2018;156(4):1611.

51. Fawzy H, Alhodaib N, Mazer CD, et al. Sternal plating for primary and secondary sternal closure; can it improve sternal stability. J Cardiothorac Surg 2009;4:19.

52. Gottlieb LJ, Pielet RW, Karp RB, et al. Rigid internal fixation of the sternum in postoperative mediastinitis. Arch Surg 1994;129(5):489-93.

53. Hirose H, Yamane K, Youdelman BA, et al. Rigid sternal fixation improves postoperative recovery. Open Cardiovasc Med J 2011;5:148-52.

54. Snyder CW, Graham LA, Byers RE, et al. Primary sternal plating to prevent sternal wound complications after cardiac surgery: early experience and patterns of failure. Interact Cardiovasc Thorac Surg 2009;9(5):763-6.

55. Matsuyama K, Kuinose M, Koizumi N, et al. Sternal closure by rigid plate fixation in off-pump coronary artery bypass grafting: a comparative study. J Artif Organs 2016;19(2):175-8.

56. Raman J, Lehmann S, Zehr K, et al. Sternal closure with rigid plate fixation versus wire closure: a randomized controlled multicenter trial. Ann Thorac Surg 2012; 94(6):1854-61.

57. Allen KB, Icke KJ, Thourani VH, et al. Sternotomy closure using rigid plate fixation: a paradigm shift from wire cerclage. Ann Cardiothorac Surg 2018;7(5): 611-20.

58. Nazerali RS, Hinchcliff K, Wong MS. Rigid fixation for the prevention and treatment of sternal complications. Ann Plast Surg 2014;72(Suppl 1):S27-30.

59. Park JS, Kuo JH, Young JN, et al. Rigid sternal fixation versus modified wire technique for poststernotomy closures: a retrospective cost analysis. Ann Plast Surg 2017;78(5):537-42.

60. Liao JM, Chan P, Cornwell L, et al. Feasibility of primary sternal plating for morbidly obese patients after cardiac surgery. J Cardiothorac Surg 2019; 14(1):25.

61. Allen KB, Thourani VH, Naka Y, et al. Rigid plate fixation versus wire cerclage: patient-reported and economic outcomes from a randomized trial. Ann Thorac Surg 2018;105(5):1344-50.

62. Tam DY, Nedadur R, Yu M, et al. Rigid plate fixation versus wire cerclage for sternotomy after cardiac surgery: a meta-analysis. Ann Thorac Surg 2018;106(1): 298-304.

63. Brown JK, Singh K, Dumitru R, et al. The benefits of enhanced recovery after surgery programs and their application in cardiothoracic surgery. Methodist Debakey Cardiovasc J 2018;14(2):77-88.

64. Krzych $€$, Kucewicz-Czech E. It is time for enhanced recovery after surgery in cardiac surgery. Kardiol Pol 2017;75(5):415-20. 
65. Ljungqvist $\mathrm{O}$. The enhanced recovery after surgery in cardiac surgery revolution. JAMA Surg 2019;154(8):767.

66. Baron S, Reynolds MR, Cohen DJ. Economic considerations for TAVR Vs. SAVR: historical perspective and future predictions. American College of Cardiology website. 2019. Available at: https://www.acc.org/latest-in-cardiology/articles/ 2019/06/18/07/43/economic-considerations-for-tavr-vs-savr. Accessed: January 10, 2020.

67. Baron SJ, Magnuson EA, Lu M, et al. Health status after transcatheter versus surgical aortic valve replacement in low-risk patients with aortic stenosis. J Am Coll Cardiol 2019;74(23):2833-42.

68. Olds A, Saadat S, Azzolini A, et al. Improved operative and recovery times with mini-thoracotomy aortic valve replacement. J Cardiothorac Surg 2019;14(1):91.

69. Stoliński J, Plicner D, Grudzień G, et al. A comparison of minimally invasive and standard aortic valve replacement. J Thorac Cardiovasc Surg 2016;152(4): 1030-9.

70. Tokarek T, Siudak Z, Dziewierz A, et al. Assessment of quality of life in patients after surgical and transcatheter aortic valve replacement. Catheter Cardiovasc Interv 2016;88(3):E80-8. 